\title{
Malonylation/Decarbalkoxylation of Furan Derivatives as Key Steps for the Preparation of Nonactic Acid Derivatives. Part II [1]
}

\author{
Jean-Mary Simone, François Loiseau, David Carcache, Pavel Bobal, \\ Julie Jeanneret-Gris, and Reinhard Neier*
}

Institute of Chemistry, University of Neuchâtel, Neuchâtel, Switzerland

Received July 17, 2006; accepted (revised) July 26, 2006; published online January 19, 2007

(C) Springer-Verlag 2007

\begin{abstract}
Summary. A malonylation/decarbalkoxylation sequence from 2-substituted furans was investigated in view of developing a scalable synthesis of hydrophobic nonactic acid analogues.
\end{abstract}

Keywords. Heterocycles; Decarbalkoxylation; Nonactic acid; Natural-like.

\section{Introduction}

The ionophore nonactin is used in ion selective sensors because of its selectivity for ammonium and potassium cations [2]. The life time of these electrodes is limited due to the loss of nonactin through bleeding. In the course of our studies for the preparation of hydrophobic nonactin derivatives [3], we described a rapid synthesis of derivatives of nonactic acid from furan applying different radical couplings for the introduction of the first aliphatic side chain [4]. In Ref. [1], we described the introduction of the second aliphatic chain starting from malonyl derivatives as reagents. This transformation could be achieved in good yields and under total conversion of our starting materials. In this paper we present our results on the second decarbalkoxylation step (Scheme 1).

\section{Results and Discussion}

We first investigated the decarbalkoxylation of our dimethyl malonates 1-3. We studied the enzymatic

\footnotetext{
* Corresponding author. E-mail: reinhard.neier@unine.ch
}

mono-hydrolysis with pig liver esterase (PLE), followed by decarboxylation [5]. Applying PLE to this reaction is justified by the soft reaction conditions which are compatible with the heat-sensitivity of the furan derivatives. The selective enzymatic monomethylester hydrolysis for the two first products $\mathbf{1}$ and 2 occurred smoothly to give $\mathbf{4}$ and $\mathbf{5}$ in 63$64 \%$ yields (Scheme 2). To achieve complete hydrolysis of 2 proved to be more difficult; $24 \mathrm{~h}$ were required instead of the $4 \mathrm{~h}$ which were sufficient for the transformation of $\mathbf{1}$. The additional steric hindrance conferred to $\mathbf{2}$ by the introduction of the additional methyl group is probably responsible for the longer reaction times needed [6]. For 4 we measured the optical rotation, indicating that the enzyme is capable of distinguishing between the two enantiotopic ester groups. The enzyme was not able to hydrolyse the hydrophobic derivative 3. After heating 4 from 100 to $200^{\circ} \mathrm{C}$ at 0.019 Torr in the Kugelrohr in order to achieve the decarboxylation, only $43 \%$ of 6 were obtained. As the heat-sensitivity of furans is well documented this result did not come as a surprise (Scheme 2).

Since the enzymatic hydrolysis was not adequate for the transformation of the hydrophobic 3, we searched for alternative methods. Krapcho [7] and Van der Gen [8] use $\mathrm{NaCl}$ in wet DMSO. This reaction was successfully applied to 3 and gave 7 in $56 \%$ yield (Scheme 3 ). Total conversion of $\mathbf{3}$ was observed. The $R_{\mathrm{f}}$ values on TLC of both 3 and 7 were almost the same. Separation 


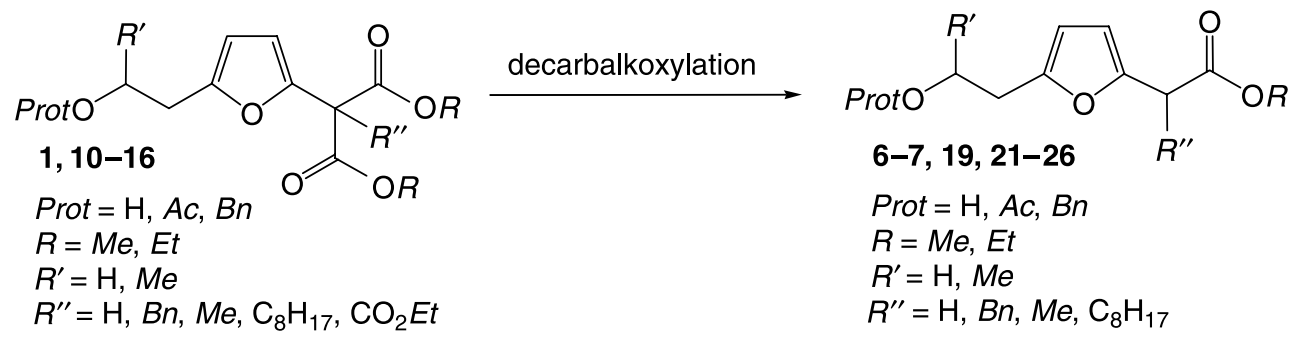

Scheme 1<smiles>[2H]C(C(=O)OC)(C(=O)OC)c1ccc(CCO)o1</smiles>

$\underset{\mathrm{pH}=7-8, \mathrm{rt}}{\stackrel{P L E, \text { phosphate buffer }}{\longrightarrow}}$<smiles>COC(=O)C(C(=O)O)(C(=O)O)c1ccc(CCO)o1</smiles>

4, $R=\mathrm{H}, 4 \mathrm{~h}, 63 \%$, $[\alpha]_{\mathrm{D}}^{20}=-6.29^{\circ} \cdot \mathrm{cm}^{2} \cdot \mathrm{g}^{-1} \cdot 10^{-1}$

5, $R=M e, 24 \mathrm{~h}, 64 \%$ $R=\mathrm{H}$, heat $43 \%$<smiles>COC(=O)Cc1ccc(CCO)o1</smiles>

Scheme 2<smiles>COC(=O)C(C)(C(=O)OC)c1ccc(CCO)o1</smiles><smiles>CCCCCCCCCCCCCCCC(C(=O)OC)c1ccc(CCO)o1</smiles>

Scheme 3

by column chromatography under the conditions studied would not have been possible. The heat sensitivity of furan derivatives can tentatively explain the loss of product once more.

To improve the yields we checked the consequence of reducing the reaction time. Under the conditions reported by Rapoport $\mathrm{NaCl}$ is replaced by $\mathrm{LiCl}$ [9]. Good yields could be obtained under these conditions heating for only $2 \mathrm{~h}$ at $190^{\circ} \mathrm{C}$ (Table 1 ). The first attempts using our model compounds $\mathbf{8}$ and 9 gave low yields of 25 and $23 \%$ of $\mathbf{1 7}$ and $\mathbf{1 8}$. To obtain these products two or four decarbethoxylations have to occur in sequence. The overall yield of such a transformation composed of several steps are by necessity relatively low even if the yield of the individual reaction is good to acceptable. We did not optimise these two transformations and focused our efforts on our target molecules 21-26. Transforming the free alcohol $\mathbf{1 0}$ under these conditions the yield of 19 was only $19 \%$. We could isolate traces of the dimer $\mathbf{2 0}$ as side product. The formation of $\mathbf{2 0}$ can be explained as a transesterification of the ethylester part of one molecule of $\mathbf{2 0}$ with the alcohol part of a second molecule of $\mathbf{1 9}$. We assumed that other heavier oligomers and/or polymers were formed by poly-transesterification of $\mathbf{1 9}$, which could explain the low yield of isolated $\mathbf{1 9}$.

To avoid side reactions, we used the O-protected molecules 11-16. Under the same reaction conditions the O-benzylated molecules 11-15 gave 21-25 with satisfactory $63-91 \%$ yields. The O-acetyl protecting group of $\mathbf{1 6}$ was not resistant enough towards the reaction conditions. In this case, the yield in $\mathbf{2 6}$ was only $4 \%$, and $14 \%$ of the deprotected product 19 
Table 1. Decarbethoxylation in Rapoport conditions, with $\mathrm{LiCl}$

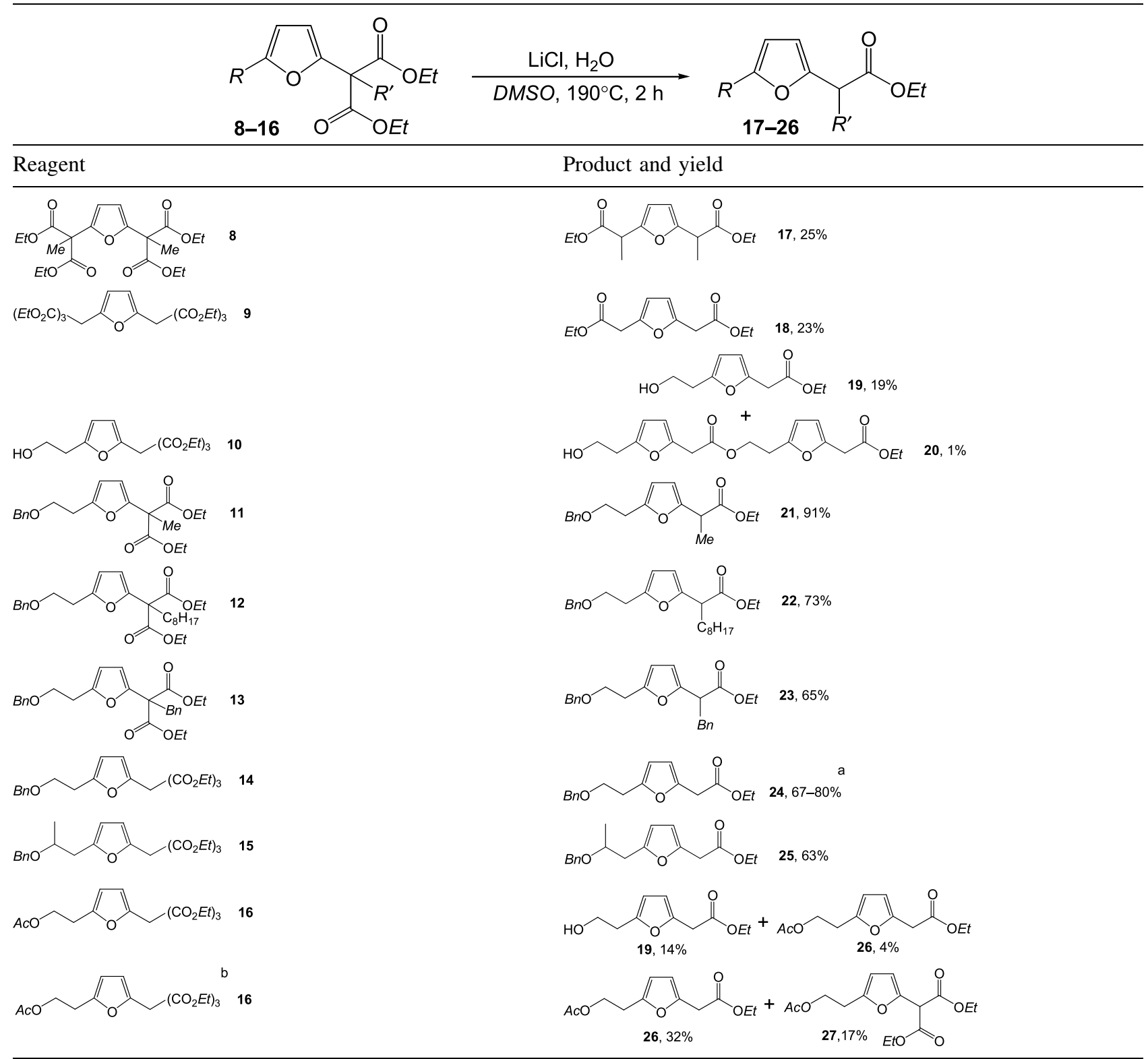

${ }^{\mathrm{a}}$ Reaction performed several times in this reproducible range of yields; ${ }^{\mathrm{b}}$ reaction performed at $160^{\circ} \mathrm{C}$ instead of $190^{\circ} \mathrm{C}$

were obtained. We then tried to reduce the reaction temperature to $160^{\circ} \mathrm{C}$ with an aim to prevent the $\mathrm{O}$ acetyl cleavage, but the reaction was not complete. At $160^{\circ} \mathrm{C}$, the yield in 26 was only $32 \%$, and we isolated $17 \%$ of 27 , where only one of the two ethoxycarbonyl groups of $\mathbf{1 6}$ was removed.

\section{Conclusions}

A set of nonactic acid derivatives precursors were successfully prepared. For the two target products $\mathbf{2 1}$ and $\mathbf{2 4}$, the best overall yields of the malonylation/ decarbalkoxylation sequence were 74 and $71 \%$. The hydrophobic precursor 12 afforded $45 \%$ of 22 over the two-step sequence. The reported yields were determined using chromatography for the purification of the products obtained after each step. The purification by chromatography is not obligatory. However, the overall yields for the two step sequence malonylation/ decarbalkoxylation were slightly better if the intermediate was purified by chromatography.

This efficient and scalable strategy will be applied for the preparation of macrocyclic analogues of the natural product nonactin. 


\section{Experimental}

All moisture-sensitive reactions were carried out under Ar and $\mathrm{N}_{2}$ using oven-dried glassware. All reagents were of commercial quality if not specifically mentioned. Solvents were freshly distilled prior to use. Flash chromatography (FC): Brunschwig silica gel $60,0.032-0.063 \mathrm{~mm}$, under positive pressure. TLC: Merck precoated silica gel thin-layer sheets $60 \mathrm{~F} 254$, detection by UV and treatment with basic $\mathrm{KMnO}_{4}$ sol. Mp: Gallenkamp MFB-595. IR spectra: Perkin Elmer Spectrum One FT-IR, in $\mathrm{cm}^{-1}$. NMR spectra: Bruker Avance$400\left(400 \mathrm{MHz}\left({ }^{1} \mathrm{H}\right)\right.$ and $\left.100 \mathrm{MHz}\left({ }^{13} \mathrm{C}\right)\right)$, at rt, chemical shifts $\delta$ in ppm rel. to $\mathrm{CDCl}_{3}\left({ }^{1} \mathrm{H}: 7.264 \mathrm{ppm},{ }^{13} \mathrm{C}: 77.0 \mathrm{ppm}\right)$ as internal reference, coupling constants $J$ in Hz. ESI-MS: Finnigan LCQ. Elemental analyses or HR-ESI-MS of novel compounds agreed favourably with calculated values.

\section{2-(5-(2-Hydroxyethyl)furan-2-yl)-3-methoxy-}

3-oxopropanoic acid $\left(4, \mathrm{C}_{10} \mathrm{H}_{12} \mathrm{O}_{6}\right)$

A mixture of diester 1 (440 mg, $1.72 \mathrm{mmol})$ and PLE (3 mg, 130 units/mg, 390 units) in $1 \mathrm{~cm}^{3} \mathrm{MeOH}$ and $10 \mathrm{~cm}^{3} \mathrm{pH}=$ 8.0 phosphate buffer was stirred at room temperature for $4 \mathrm{~h}$. During the hydrolysis, $0.1 \mathrm{M} \mathrm{NaOH}$ solution was periodically added to maintain the $p H$ of the solution at 7-8 (total $17.2 \mathrm{~cm}^{3}$ $0.1 M \mathrm{NaOH})$. The aqueous layer was basified to $p H=9.0$ by the addition of more $\mathrm{NaOH}$ solution and washed 5 times with diethyl ether. After the enzyme was filtered off, the aqueous layer was acidified to $p H<3$ with $1 \mathrm{M} \mathrm{HCl}$ solution, and the products were extracted 5 times with diethyl ether, dried $\left(\mathrm{Na}_{2} \mathrm{SO}_{4}\right)$, and the solvent was evaporated in vacuo. Separation by chromatography afforded unreacted starting material 1 (44 mg, $0.18 \mathrm{mmol}, 15 \%)$ and $4(245 \mathrm{mg}, 1.07 \mathrm{mmol}, 63 \%)$ as a colorless oil. $R_{\mathrm{f}}=0.22\left(\mathrm{CH}_{2} \mathrm{Cl}_{2} / E t_{2} \mathrm{O} / E t \mathrm{OH}=8 / 2 / 1\right)$; IR (film): $\bar{\nu}=3475,2958,2602,1741,1560,1439,1391$, $1278,1231,1161,1020 \mathrm{~cm}^{-1} ;{ }^{1} \mathrm{H} \mathrm{NMR}\left(400 \mathrm{MHz}, \mathrm{CDCl}_{3}\right)$ : $\delta=6.35(\mathrm{~d}, J=3.2 \mathrm{~Hz}, \mathrm{H}-4), 6.10(\mathrm{~d}, J=3.2 \mathrm{~Hz}, \mathrm{H}-5), 4.77$ (s, H-2), $4.75(b r, \mathrm{OH}), 3.87(\mathrm{t}, J=6.2 \mathrm{~Hz}, \mathrm{H}-8), 3.78(\mathrm{~s}$, $\left.\mathrm{H}-1^{1}\right), 2.88(\mathrm{t}, J=6.2 \mathrm{~Hz}, \mathrm{H}-7), \mathrm{ppm} ;{ }^{13} \mathrm{C} \mathrm{NMR}(100 \mathrm{MHz}$, $\left.\mathrm{CDCl}_{3}\right): \delta=169.5(C=\mathrm{O}), 167.0(C=\mathrm{O}), 153.6(\mathrm{C}-3), 144.0$ (C-6), 110.5 (C-4), 107.8 (C-5), 60.9 (C-8), $53.3\left(\right.$ C- $\left.^{1}{ }^{1}\right), 51.4$ (C-2), 31.4 (C-7) ppm; EI-MS: $m / z=227$ (traces, $[\mathrm{M}-\mathrm{H}]^{+}$), $184\left(37,\left[\mathrm{M}-\mathrm{CO}_{2}\right]^{+}\right), 154$ (35), 153 (35), 111 (76), 106 (47), 95 (52), 94 (100), 65 (38).

\section{2-(5-(2-Hydroxyethyl)furan-2-yl)-3-methoxy-}

2-methyl-3-oxopropanoic acid $\left(\mathbf{5}, \mathrm{C}_{11} \mathrm{H}_{14} \mathrm{O}_{6}\right)$

A mixture of diester $2(270 \mathrm{mg}, 1.07 \mathrm{mmol})$ and PLE $(2.23 \mathrm{mg}$, 220 units $/ \mathrm{mg}, 490$ units) in $0.5 \mathrm{~cm}^{3} \mathrm{MeOH}$ and $7 \mathrm{~cm}^{3} \mathrm{pH}=$ 8.0 phosphate buffer was stirred at room temperature for $24 \mathrm{~h}$. During the hydrolysis, $0.1 \mathrm{M} \mathrm{NaOH}$ solution was periodically added to maintain the $p H$ of the solution at $7-8\left(\right.$ total $10.7 \mathrm{~cm}^{3}$

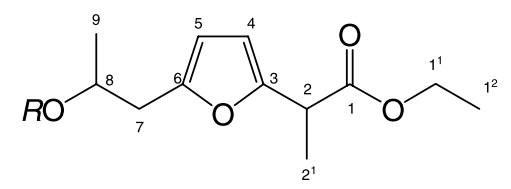

Fig. 1. Labeling used for NMR assignment
$0.1 M \mathrm{NaOH})$. The aqueous layer was basified to $p H=9.0$ by the addition of more $\mathrm{NaOH}$ solution and washed with brine, the aqueous layer was then acidified to $p H=1$ with $1 \mathrm{M} \mathrm{HCl}$ solution, and extracted 5 times with diethyl ether, dried $\left(\mathrm{Na}_{2} \mathrm{SO}_{4}\right)$, and the solvent was evaporated in vacuo to afford 5 (165 mg, $0.68 \mathrm{mmol}, 64 \%)$. Oil; $R_{\mathrm{f}}=0.46\left(E t_{2} \mathrm{O} / \mathrm{MeOH}=\right.$ 50/50); IR (film): $\bar{\nu}=3474,2999,2955,2612,1960,1730$, $1610,1555,1456,1437,1380,1250,1125,1110,1032 \mathrm{~cm}^{-1}$; ${ }^{1} \mathrm{H}$ NMR $\left(400 \mathrm{MHz}, \mathrm{CDCl}_{3}\right): \delta=6.30(\mathrm{~d}, J=3.2 \mathrm{~Hz}, \mathrm{H}-4)$, $6.10(\mathrm{~d}, J=3.2 \mathrm{~Hz}, \mathrm{H}-5), 5.97(b r, \mathrm{OH}), 3.87(\mathrm{t}, J=6.2 \mathrm{~Hz}$, H-8), 3.81 (s, H-1 $\left.{ }^{1}\right), 2.89$ (t, $\left.J=6.2 \mathrm{~Hz}, \mathrm{H}-7\right), 1.85$ (s, H-2 $\left.{ }^{1}\right)$ ppm; ${ }^{13} \mathrm{C}$ NMR $\left(100 \mathrm{MHz}, \mathrm{CDCl}_{3}\right): \delta=173.3(C=\mathrm{O}), 170.2$ $(C=\mathrm{O}), 153.1$ (C-3), 148.9 (C-6), 108.9 (C-4), 107.3 (C-5), 60.9 (C-8), 54.7 (C-2), $53.2\left(\mathrm{C}-1^{1}\right), 31.2(\mathrm{C}-7), 20.1\left(\left(\mathrm{C}-2^{1}\right)\right.$ ppm; EI-MS: $m / z=198\left(34,\left[\mathrm{M}-\mathrm{CO}_{2}\right]^{+}\right), 181$ (13), 180 (21, $\left.\left[\mathrm{M}-\mathrm{CO}_{2}-\mathrm{H}_{2} \mathrm{O}\right]^{+}\right), 168$ (44), 167 (31), 166 (11), 140 (12), 139 (100), 135 (16), 121 (76), 111 (38), 109 (20), 108 (30), 107 (14), 81 (44), 80 (21), 79 (24), 77 (17), 65 (12), 45 (20); ESI-HR-MS: $m / z[\mathrm{M}+\mathrm{Na}]^{+}=$calcd 265.0683, found 265.0682 .

\section{Methyl 2-(5-(2-hydroxyethyl)furan-2-yl)acetate}

$\left(6, \mathrm{C}_{9} \mathrm{H}_{12} \mathrm{O}_{4}\right)$

Distillation of $4(129 \mathrm{mg}, 0.565 \mathrm{mmol})$ in a Kugelrohr apparatus from 100 to $200^{\circ} \mathrm{C}$ at 0.019 Torr afforded $6(45 \mathrm{mg}, 0.244 \mathrm{mmol}$, $43 \%)$. Oil; $R_{\mathrm{f}}=0.26 \quad\left(\mathrm{CH}_{2} \mathrm{Cl}_{2} / E t_{2} \mathrm{O}=75 / 25\right)$; IR (film): $\bar{\nu}=3136,2956,1742,1615,1566,1439,1406,1337,1275$, $1228,1198,1158,1015 \mathrm{~cm}^{-1} ;{ }^{1} \mathrm{H}$ NMR $\left(400 \mathrm{MHz}, \mathrm{CDCl}_{3}\right)$ : $\delta=6.10(\mathrm{~d}, J=3.1 \mathrm{~Hz}, \mathrm{H}-4), 6.02(\mathrm{~d}, J=3.1 \mathrm{~Hz}, \mathrm{H}-5), 3.81$ $(\mathrm{t}, J=6.3 \mathrm{~Hz}, \mathrm{H}-8), 3.69\left(\mathrm{~s}, \mathrm{H}-1^{1}\right), 3.62(\mathrm{~s}, \mathrm{H}-2), 2.82(\mathrm{t}$, $J=6.3 \mathrm{~Hz}, \mathrm{H}-7), 2.18(\mathrm{br}, \mathrm{OH}) \mathrm{ppm} ;{ }^{13} \mathrm{C} \mathrm{NMR}(100 \mathrm{MHz}$, $\left.\mathrm{CDCl}_{3}\right): \delta=170.1(C=\mathrm{O}), 152.5,146.3(\mathrm{C}-3, \mathrm{C}-6), 108.8$ (C-4), 107.4 (C-5), 60.9 (C-8), $53.0\left(\mathrm{C}-1^{1}\right), 33.8$ (C-2), 31.6 (C-7) ppm; EI-MS: $m / z=184\left(26,[\mathrm{M}]^{+}\right), 154$ (26), 153 (22, $\left.\left[\mathrm{M}-\mathrm{CH}_{3} \mathrm{O}\right]^{+}\right), 125\left(32,\left[\mathrm{M}-\mathrm{CH}_{3} \mathrm{OCO}\right]^{+}\right), 111(73), 107$ (33), 95 (55), 94 (100), 81 (41), 65 (47).

\section{General Procedure for Decarbalkoxylation}

A solution of the triester or the malonate $(1 \mathrm{eq}), \mathrm{NaCl}$ or $\mathrm{LiCl}$ (1-10 eq), and $\mathrm{H}_{2} \mathrm{O}$ (1-3 eq) in DMSO was heated at 160$190^{\circ} \mathrm{C}$ for several $\mathrm{h}$. After cooling at $\mathrm{rt}$, the product was extracted with diethyl ether or AcOEt. The extract was washed with brine and dried $\left(\mathrm{Na}_{2} \mathrm{SO}_{4}\right)$. Evaporation of the solvent in vacuo afforded the product, which was purified by chromatography on a silica gel column if necessary.

\section{Methyl 2-(5-(2-hydroxyethyl)furan-2-yl)decanoate}

$\left(3, \mathrm{C}_{17} \mathrm{H}_{28} \mathrm{O}_{4}\right)$

General procedure with $7(1.29 \mathrm{~g}, 3.6 \mathrm{mmol}), \mathrm{NaCl}(0.21 \mathrm{~g}$, $3.6 \mathrm{mmol})$, and $\mathrm{H}_{2} \mathrm{O}(0.13 \mathrm{~g}, 7.2 \mathrm{mmol})$ in $45 \mathrm{~cm}^{3}$ DMSO heated at $160^{\circ} \mathrm{C}$ for $3.5 \mathrm{~h}$. Chromatography afforded $7(0.60 \mathrm{~g}$, $2.03 \mathrm{mmol}, 56 \%)$. Oil; $R_{\mathrm{f}}=0.29\left(\mathrm{CH}_{2} \mathrm{Cl}_{2} / E t_{2} \mathrm{O}=75 / 25\right)$; IR (film): $\bar{\nu}=3108,2954,2928,2857,1742,1610,1561$, $1461,1436,1379,1212,1161,1121,1050 \mathrm{~cm}^{-1} ;{ }^{1} \mathrm{H}$ NMR $\left(400 \mathrm{MHz}, \mathrm{CDCl}_{3}\right): \delta=6.08(\mathrm{~d}, J=3.1 \mathrm{~Hz}, \mathrm{H}-4), 6.03(\mathrm{~d}$, $J=3.1 \mathrm{~Hz}, \mathrm{H}-5), 3.84(\mathrm{t}, J=6.2 \mathrm{~Hz}, \mathrm{H}-8), 3.69\left(s, \mathrm{H}-1^{1}\right)$, $2.86(\mathrm{t}, J=6.2 \mathrm{~Hz}, \mathrm{H}-7), 1.98-1.85$ (m, H-2 $\left.{ }^{1}\right), 1.77$ (br, OH), $1.29-1.15\left(\mathrm{~m}, \mathrm{H}-2^{2}\right.$ to $\left.\mathrm{H}-2^{7}\right), 0.87\left(\mathrm{t}, J=6.7 \mathrm{~Hz}, \mathrm{H}-2^{8}\right) \mathrm{ppm}$; 
${ }^{13} \mathrm{C} \mathrm{NMR}\left(100 \mathrm{MHz}, \mathrm{CDCl}_{3}\right): \delta=172.7(C=\mathrm{O}), 152.0(\mathrm{C}-3)$, 151.3 (C-6), 107.2 (C-4), 105.8 (C-5), $61.0(\mathrm{C}-8), 52.1\left(\mathrm{C}-1^{1}\right)$, 45.6 (C-2), $31.6(\mathrm{C}-7), 30.9 \mathrm{C}\left(2^{1}\right), 31.8,29.3,29.2,29.1,27.3$, $22.6\left(\mathrm{C}-2^{2}\right.$ to $\left.\mathrm{C}-2^{7}\right), 14.0\left(\mathrm{C}-2^{8}\right)$ ppm; EI-MS: $m / z=296(15$, $\left.[\mathrm{M}]^{+}\right), 267$ (11), $266(46), 265\left(14,\left[\mathrm{M}-\mathrm{CH}_{3} \mathrm{O}\right]^{+}\right), 238$ (13), $237\left(75,\left[\mathrm{M}-\mathrm{CH}_{3} \mathrm{OCO}\right]^{+}\right), 219$ (21), 208 (21), 207 (100), 205 (11), 191 (10), 183 (13), 165 (15), 153 (42), 152 (11), 149 (11), 125 (52), 121 (40), 111 (25), 107 (47), 95 (30), 94 (14), 91 (11), 86 (15), 84 (24), 83 (20), 82 (16), 81 (69), 80 (30), 79 (23), 77 (20), 73 (39), 71 (17), 69 (19), 67 (13), 65 (11), 55 (18), 44 (12); ESI-HR-MS: $m / z[\mathrm{M}+\mathrm{Na}]^{+}=$calcd 319.1880 , found 319.1885 .

\section{Ethyl 2-(5-(1-ethoxycarbonyl-ethyl)-furan-2-yl)propionate}

$\left(17, \mathrm{C}_{14} \mathrm{H}_{20} \mathrm{O}_{5}\right)$

General procedure with 8 (700 mg, $1.70 \mathrm{mmol}), \mathrm{LiCl}(0.44 \mathrm{~g}$, $10.4 \mathrm{mmol})$, and $\mathrm{H}_{2} \mathrm{O}(60 \mathrm{mg}, 3.3 \mathrm{mmol})$ in $3 \mathrm{~cm}^{3} \mathrm{DMSO}$ heated at $190^{\circ} \mathrm{C}$ for $2 \mathrm{~h}$. Chromatography afforded $\mathbf{1 7}$ (113 mg, $4.2 \mathrm{mmol}, 25 \%)$. Oil; $R_{\mathrm{f}}=0.35$ ( $n$-hexane $/ A c \mathrm{OEt}=$ $75 / 25+1 \% \mathrm{MeOH}$ ); IR (film): $\bar{\nu}=2985,2942,2908,2883$, 1738, 1643, 1607, 1457, 1377, 1320, 1252, 1204, 1162, 1095, $1072,1025 \mathrm{~cm}^{-1} ;{ }^{1} \mathrm{H}$ NMR $\left(400 \mathrm{MHz}, \mathrm{CDCl}_{3}\right): \delta=6.060$ and 6.057 (2s, H-4 rac and H-4 meso), 4.12 (q, $J=7.1 \mathrm{~Hz}, \mathrm{H}-1^{1}$ ), $3.72(\mathrm{q}, J=7.3 \mathrm{~Hz}, \mathrm{H}-2), 1.45\left(\mathrm{~d}, J=7.3 \mathrm{~Hz}, \mathrm{H}-2^{1}\right), 1.20$ $\left(\mathrm{t}, J=7.1 \mathrm{~Hz}, \mathrm{H}-1^{2}\right) \mathrm{ppm} ;{ }^{13} \mathrm{C} \mathrm{NMR}\left(100 \mathrm{MHz}, \mathrm{CDCl}_{3}\right)$ : $\delta=172.42$ and $172.46(C=\mathrm{O}$ rac and $C=\mathrm{O}$ meso $), 152.46$ and 152.41 (C-3 rac and C-3 meso), 106.4 (C-4 rac, C-4 meso), $60.8\left(\mathrm{C}-1^{1}\right.$ rac, $\mathrm{C}-1^{1}$ meso $), 39.4$ (C-2 rac, C-2 meso), 15.57 and $15.61\left(\mathrm{C}-2^{1} \mathrm{rac}\right.$ and $\mathrm{C}-2^{1}$ meso $), 14.0\left(\mathrm{C}-1^{2} \mathrm{rac}, \mathrm{C}-1^{2}\right.$ meso) ppm; EI-MS: $m / z=269\left(3, \quad[\mathrm{M}+\mathrm{H}]^{+}\right), \quad 268$ $\left(14,[\mathrm{M}]^{+}\right), 196\left(11,\left[\mathrm{M}+\mathrm{H}-\mathrm{CO}_{2} \mathrm{C}_{2} \mathrm{H}_{5}\right]^{+}\right), 195$ (100, $\left[\mathrm{M}-\mathrm{CO}_{2} \mathrm{C}_{2} \mathrm{H}_{5}\right]^{+}$), 139 (11), 122 (22), 121 (18), 111 (22), 107 (27); ESI-HR-MS: $m / z[\mathrm{M}+\mathrm{Na}]^{+}=$calcd 291.1203, found 291.1202 .

\section{Ethyl (5-ethoxycarbonyl-methyl-furan-2-yl)acetate}

(18, $\mathrm{C}_{12} \mathrm{H}_{16} \mathrm{O}_{5}$ )

General procedure with 9 (4.27 g, $8.08 \mathrm{mmol}), \mathrm{LiCl}(2.05 \mathrm{~g}$, $48.5 \mathrm{mmol})$, and $\mathrm{H}_{2} \mathrm{O}(315 \mathrm{mg}, 17.5 \mathrm{mmol})$ in $20 \mathrm{~cm}^{3}$ DMSO heated at $190^{\circ} \mathrm{C}$ for $2 \mathrm{~h}$. Chromatography afforded $18(442 \mathrm{mg}$, $1.84 \mathrm{mmol}, 23 \%)$. Oil; $R_{\mathrm{f}}=0.24$ ( $n$-hexane/ $A c \mathrm{OE} t=75 /$ $25+1 \% \mathrm{MeOH}$ ); IR (film): $\bar{\nu}=2984,2939 ; 2908,1742$, 1649, 1617, 1566, 1466, 1447, 1396, 1370, 1338, 1302, 1266, 1224, 1181, 1097, $1032 \mathrm{~cm}^{-1} ;{ }^{1} \mathrm{H}$ NMR (400 MHz, $\left.\mathrm{CDCl}_{3}\right)$ : $\delta=6.18(\mathrm{~s}, \mathrm{H}-4), 4.19\left(\mathrm{q}, J=7.1 \mathrm{~Hz}, \mathrm{H}-1^{1}\right), 3.66(\mathrm{~s}, \mathrm{H}-2)$, $1.29\left(\mathrm{t}, J=7.1 \mathrm{~Hz}, \mathrm{H}-1^{2}\right) \mathrm{ppm} ;{ }^{13} \mathrm{C} \mathrm{NMR}\left(100 \mathrm{MHz}, \mathrm{CDCl}_{3}\right)$ : $\delta=169.8(C=\mathrm{O}), 147.7(\mathrm{C}-3), 109.3(\mathrm{C}-4), 61.5\left(\mathrm{C}-1^{1}\right), 34.6$ (C-2), $14.6\left({\left.\mathrm{C}-1^{2}\right)}^{2}\right.$ ppm; EI-MS: $m / z=263\left(17,\left[\mathrm{M}+\mathrm{Na}^{+}\right)\right.$, 260 (20), $258\left(100,\left[\mathrm{M}+\mathrm{H}_{2} \mathrm{O}\right]^{+}\right), 243$ (15), 241 (16), 241 (10).

\section{Ethyl 2-(5-(2-hydroxyethyl)furan-2-yl)acetate}

$\left(\mathbf{1 9}, \mathrm{C}_{10} \mathrm{H}_{14} \mathrm{O}_{4}\right)$ and 2-(5-Ethoxycarbonylmethyl

furan-2-yl)ethyl (5-(2-hydroxyethyl)furan-2-yl)-3-acatate

(20, $\mathrm{C}_{18} \mathrm{H}_{22} \mathrm{O}_{7}$ )

General procedure with $\mathbf{1 0}(1.44 \mathrm{~g}, 4.22 \mathrm{mmol}), \mathrm{LiCl}(0.55 \mathrm{~g}$, $13.0 \mathrm{mmol})$, and $\mathrm{H}_{2} \mathrm{O}(80 \mathrm{mg}, 4.4 \mathrm{mmol})$ in $11 \mathrm{~cm}^{3}$ DMSO heated at $190^{\circ} \mathrm{C}$ for $2 \mathrm{~h}$. Chromatography afforded 19 (160 mg, $0.808 \mathrm{mmol}, 19 \%)$ and $\mathbf{2 0}$ (18.4 mg, $0.053 \mathrm{mmol}, 1 \%$ ).
19: Data were the same than under Baciocchi conditions. See data reported in the previous paper [4].

20: Oil; $R_{\mathrm{f}}=0.06(n$-hexane $/ A c \mathrm{OEt}=75 / 25+1 \% \mathrm{MeOH})$; ${ }^{1} \mathrm{H} \mathrm{NMR}\left(400 \mathrm{MHz}, \mathrm{CDCl}_{3}\right): \delta=6.13(\mathrm{~d}, J=3.1 \mathrm{~Hz}, \mathrm{H}-4$, $\left.\mathrm{H}-8^{4}\right), 6.06$ and $6.01\left(2 \mathrm{~d}, J=3.1 \mathrm{~Hz}, \mathrm{H}-5\right.$ and $\left.\mathrm{H}-8^{5}\right), 4.36$ $(\mathrm{t}, J=6.8 \mathrm{~Hz}, \mathrm{H}-8), 4.20\left(\mathrm{q}, J=7.1 \mathrm{~Hz}, \mathrm{H}-1^{1}\right), 3.86(\mathrm{t}$, $\left.J=6.2 \mathrm{~Hz}, \mathrm{H}-8^{8}\right), 3.66$ and $3.64\left(2 \mathrm{~s}, \mathrm{H}-2\right.$ and $\left.\mathrm{H}-8^{2}\right), 2.97(\mathrm{t}$, $J=6.8 \mathrm{~Hz}, \mathrm{H}-7), 2.87\left(\mathrm{t}, J=6.2 \mathrm{~Hz}, \mathrm{H}-8^{7}\right), 1.77$ (br, OH), $1.29\left(\mathrm{t}, J=7.1 \mathrm{~Hz}, \mathrm{H}-1^{2}\right) \mathrm{ppm} ;{ }^{13} \mathrm{C} \mathrm{NMR}\left(100 \mathrm{MHz}, \mathrm{CDCl}_{3}\right)$ : $\delta=170.0(C=\mathrm{O}), 169.8(C=\mathrm{O}), 152.8,151.5,147.1,146.8$ $\left(\mathrm{C}-3, \mathrm{C}-8^{3}, \mathrm{C}-6, \mathrm{C}-8^{6}\right), 109.2,109.1\left(\mathrm{C}-4, \mathrm{C}-8^{4}\right), 107.9,107.8$ $\left(\mathrm{C}-5, \mathrm{C}-8^{5}\right), 63.4,61.6\left(\mathrm{C}-8, \mathrm{C}-8^{8}\right), 61.5\left(\mathrm{C}-1^{1}\right), 34.6,34.5$ $\left(\mathrm{C}-2, \mathrm{C}-8^{2}\right), 32.0,28.1\left(\mathrm{C}-7, \mathrm{C}-8^{7}\right), 14.6\left(\mathrm{C}-1^{2}\right)$ ppm; EI-MS: $m / z=553$ (23), 525 (3), 473 (10), 445 (32), 389 (35), 373 $\left(100,[\mathrm{M}+\mathrm{Na}]^{+}\right)$.

\section{Ethyl 2-(5-(2-(benzyloxy)ethyl)furan-2-yl)propanoate $\left(21, \mathrm{C}_{18} \mathrm{H}_{22} \mathrm{O}_{4}\right)$}

General procedure with $\mathbf{1 1}$ (300 mg, $0.8 \mathrm{mmol}), \mathrm{LiCl}(410 \mathrm{mg}$, $0.97 \mathrm{mmol}$ ), and $\mathrm{H}_{2} \mathrm{O}(44 \mathrm{mg}, 0.48 \mathrm{mmol})$ in $1.5 \mathrm{~cm}^{3}$ DMSO heated at $190^{\circ} \mathrm{C}$ for $2 \mathrm{~h}$. Chromatography afforded 21 (220 mg, $\quad 0.73 \mathrm{mmol}, \quad 91 \%) . \quad$ Oil; $\quad R_{\mathrm{f}}=0.23 \quad$ ( $n$-hexane/ $A c \mathrm{OEt}=75 / 25$ ); IR (film): $\bar{\nu}=3088,3063,3030,2982$, 2937, 2863, 1958, 173, 1640, 1561, 1496, 1454, 1366, 1202, 1178, 1100, 1027, 736, $698 \mathrm{~cm}^{-1}$; ${ }^{1} \mathrm{H}$ NMR $(400 \mathrm{MHz}$, $\left.\mathrm{CDCl}_{3}\right): \delta=7.39-7.28(\mathrm{~m}, P h), 6.09(\mathrm{dd}, J=3.1,0.5 \mathrm{~Hz}$, H-4), 6.02 (d, J=3.1 Hz, H-5), 4.55 (s, CH $\mathrm{CH}_{2}-\mathrm{Ph}$ ), 4.17 (q, $\left.J=7.1 \mathrm{~Hz}, \mathrm{H}-1^{1}\right), 3.77(\mathrm{q}, J=7.3 \mathrm{~Hz}, \mathrm{H}-2), 3.73$ (t, $J=$ $6.9 \mathrm{~Hz}, \mathrm{H}-8), 2.94$ (t, $J=6.9 \mathrm{~Hz}, \mathrm{H}-7), 1.50$ (d, $J=7.3 \mathrm{~Hz}$, $\left.\mathrm{H}-2^{1}\right), 1.26\left(\mathrm{t}, J=7.1 \mathrm{~Hz}, \mathrm{H}-1^{2}\right) \mathrm{ppm} ;{ }^{13} \mathrm{C} \mathrm{NMR}(100 \mathrm{MHz}$, $\left.\mathrm{CDCl}_{3}\right): \delta=173.2(C=\mathrm{O}), 152.6(\mathrm{C}-3), 152.3(\mathrm{C}-6), 138.7$ (1C, Ph), 128.8, 127.8, $128.0(5 \mathrm{C}, \mathrm{Ph}), 107.1$ (C-4), 106.9 (C-5), $73.4\left(\mathrm{CH}_{2}-\mathrm{Ph}\right), 68.7(\mathrm{C}-8), 63.4\left(\mathrm{C}-1^{1}\right), 39.9(\mathrm{C}-2)$, 29.3 (C-7), $16.2 \mathrm{C}\left(2^{1}\right), 14.6\left(\mathrm{C}-1^{2}\right)$ ppm; EI-MS: $m / z=302$ $\left(10,[\mathrm{M}]^{+}\right), 257$ (20), 229 (30), 199 (15), 181 (100), 125.23 (46), 122.30 (33), 97.47 (50), 91.49 (80), 77.39 (22).

\section{Ethyl 2-(5-(2-(benzyloxy)ethyl)furan-2-yl)decanoate}

$\left(22, \mathrm{C}_{25} \mathrm{H}_{36} \mathrm{O}_{4}\right)$

General procedure with $12(2.82 \mathrm{~g}, 6.0 \mathrm{mmol}), \mathrm{LiCl}(267 \mathrm{mg}$, $6.3 \mathrm{mmol})$, and $\mathrm{H}_{2} \mathrm{O}(337 \mathrm{mg}, 18.7 \mathrm{mmol})$ in $9 \mathrm{~cm}^{3} D M S O$ heated at $190^{\circ} \mathrm{C}$ for $2 \mathrm{~h}$. Chromatography afforded 22 $(1.76 \mathrm{~g}, 4.4 \mathrm{mmol}, 73 \%)$. Oil; $R_{\mathrm{f}}=0.42(n$-hexane $/ A c \mathrm{O} E t=$ $75 / 25+1 \% \mathrm{MeOH}$ ); IR (film): $\bar{\nu}=3030,2954,2927$, 2857, 1950, 1874, 1738, 1610, 1561, 1455, 1367, 1334, $1299,1234,1202,1176,1154,1113,1029,1016 \mathrm{~cm}^{-1} ;{ }^{1} \mathrm{H}$ NMR $\left(400 \mathrm{MHz}, \mathrm{CDCl}_{3}\right): \delta=7.38-7.26(\mathrm{~m}, P h), 6.07(\mathrm{~d}$, $J=3.2 \mathrm{~Hz}, \mathrm{H}-4), 6.00(\mathrm{~d}, J=3.2 \mathrm{~Hz}, \mathrm{H}-5), 4.53$ (s, $\mathrm{CH}_{2}-$ $P h), 4.18-4.12\left(\mathrm{~m}, J=7.1 \mathrm{~Hz}, \mathrm{H}-1^{1}, \mathrm{ABX}_{3}\right.$ system not fully resolved), $3.71(\mathrm{t}, J=6.9 \mathrm{~Hz}, \mathrm{H}-8), 2.92(\mathrm{td}, J=6.9,0.5 \mathrm{~Hz}$, H-7), 1.99-1.93 (m, H-2 $\left.{ }^{1 a}\right), 1.86-1.81\left(\mathrm{~m}, \mathrm{H}-2^{1 b}\right), 1.29-1.22$ $\left(\mathrm{m}, \mathrm{H}-2^{2}\right.$ to $\left.\mathrm{H}-2^{7}, \mathrm{H}-1^{2}\right), 0.88\left(\mathrm{t}, J=6.9 \mathrm{~Hz}, \mathrm{H}-2^{8}\right) \mathrm{ppm} ;{ }^{13} \mathrm{C}$ NMR $\left(100 \mathrm{MHz}, \mathrm{CDCl}_{3}\right): \delta=172.3(C=\mathrm{O}), 152.1 \quad(\mathrm{C}-3)$, 151.0 (C-6), 138.2 (1C, Ph), 128.3, 127.6, 127.5 (5C, Ph), 107.0 (C-4), 106.6 (C-5), $72.9\left(\mathrm{CH}_{2}-\mathrm{Ph}\right), 68.3$ (C-8), 60.8 $\left(\mathrm{C}-1^{1}\right), 45.4(\mathrm{C}-2), 31.0 \mathrm{C}\left(2^{1}\right), 31.8,29.3,29.2$ (2C), 28.9, 27.3, $22.6\left(\mathrm{C}-2^{2}\right.$ to $\left.\mathrm{C}-2^{7}, \mathrm{C}-7\right), 14.1\left(\mathrm{C}-1^{2}\right), 14.0\left(\mathrm{C}-2^{8}\right)$ ppm; ESI-MS: $m / z=424\left(14,[\mathrm{M}+\mathrm{H}+\mathrm{Na}]^{+}\right), 419(27$, $\left.\left[\mathrm{M}+\mathrm{H}+\mathrm{H}_{2} \mathrm{O}\right]^{+}\right), 418\left(98,\left[\mathrm{M}+\mathrm{H}_{2} \mathrm{O}\right]^{+}\right), 405(26), 401(100$, 
$\left.[\mathrm{M}+\mathrm{H}]^{+}\right), 327(31)$; ESI-HR-MS: $m / z[\mathrm{M}+\mathrm{H}]^{+}=$calcd 401.2686, found 401.2681.

Ethyl 2-(5-(2-(benzyloxy)ethyl)furan-2-yl)-3-

phenylpropanoate $\left(23, \mathrm{C}_{24} \mathrm{H}_{26} \mathrm{O}_{4}\right)$

General procedure with $\mathbf{1 3}(2.40 \mathrm{~g}, 5.3 \mathrm{mmol}), \mathrm{LiCl}(730 \mathrm{mg}$, $17.2 \mathrm{mmol}$ ), and $\mathrm{H}_{2} \mathrm{O}(347 \mathrm{mg}, 19.3 \mathrm{mmol})$ in $10 \mathrm{~cm}^{3} D M S O$ heated at $190^{\circ} \mathrm{C}$ for $2 \mathrm{~h}$. Chromatography afforded $23(1.30 \mathrm{~g}$, $3.4 \mathrm{mmol}, \quad 65 \%) . \quad$ Oil; $\quad R_{\mathrm{f}}=0.40 \quad(n$-hexane $/ A c \mathrm{O} E t=75 /$ $25+1 \% \mathrm{MeOH}$ ); IR (film): $\bar{\nu}=3030,2980,2958,2929$, $2861,1951,1880,1809,1736,1605,1496,1455,1367$, $1333,1275,1211,1150,1101,1080,1029,1016,699$, $404 \mathrm{~cm}^{-1} ;{ }^{1} \mathrm{H}$ NMR $\left(400 \mathrm{MHz}, \mathrm{CDCl}_{3}\right): \delta=7.39-7.16(\mathrm{~m}$, $P h), 6.10(\mathrm{~d}, J=3.0 \mathrm{~Hz}, \mathrm{H}-4), 6.02(\mathrm{~d}, J=3.0 \mathrm{~Hz}, \mathrm{H}-5)$, 4.56 (s, $\left.\mathrm{CH}_{2}-P h\right), 4.11$ (q, $\left.J=7.1 \mathrm{~Hz}, \mathrm{H}-1^{1}\right), 3.99-3.93$ (m, $\mathrm{H}-2), 3.73(\mathrm{t}, J=6.9 \mathrm{~Hz}, \mathrm{H}-8), 3.31(\mathrm{dd}, J=13.7,8.6 \mathrm{~Hz}$, $\left.\mathrm{H}-2^{1 a}\right), 3.19\left(\mathrm{dd}, J=13.7,7.2 \mathrm{~Hz}, \mathrm{H}-2^{1 b}\right), 2.96(\mathrm{t}, J=6.9 \mathrm{~Hz}$, $\mathrm{H}-7), 1.17$ (t, $\left.J=7.1 \mathrm{~Hz}, \mathrm{H}-1^{2}\right)$ ppm; ${ }^{13} \mathrm{C} \mathrm{NMR}(100 \mathrm{MHz}$, $\left.\mathrm{CDCl}_{3}\right): \delta=171.4(C=\mathrm{O}), 152.3(\mathrm{C}-3), 149.9(\mathrm{C}-6), 138.5$, $138.2,128.8,128.3,128.2,127.6,127.5,126.4(12 \mathrm{C}, P h)$, 107.6, 106.8 (2C, C-4, C-5), $72.9\left(\mathrm{CH}_{2}-\mathrm{Ph}\right), 68.2(\mathrm{C}-8)$, $60.9\left(\mathrm{C}-1^{1}\right), 37.1\left(\mathrm{C}-2^{1}\right), 28.8(\mathrm{C}-7), 14.0\left(\mathrm{C}-1^{2}\right) \mathrm{ppm}$; ESI-MS: $m / z=449$ (15), 449 (11), 433 (32), 418 (16), 417 (61), $402(26), 401\left(100,[\mathrm{M}+\mathrm{Na}]^{+}\right)$; ESI-HR-MS: $m / z$ $[\mathrm{M}+\mathrm{Na}]^{+}=$calcd 401.1729 , found 401.1722 .

\section{Ethyl 2-(5-(2-(benzyloxy)ethyl)furan-2-yl)acetate}

\section{(24, $\mathrm{C}_{17} \mathrm{H}_{20} \mathrm{O}_{4}$ )}

General procedure with $\mathbf{1 4}(2.42 \mathrm{~g}, 5.6 \mathrm{mmol}), \mathrm{LiCl}(730 \mathrm{mg}$, $17.2 \mathrm{mmol})$, and $\mathrm{H}_{2} \mathrm{O}(100 \mathrm{mg}, 5.6 \mathrm{mmol})$ in $10 \mathrm{~cm}^{3} D M S O$ heated at $190^{\circ} \mathrm{C}$ for $2 \mathrm{~h}$. Chromatography afforded $24(1.08 \mathrm{~g}$, $3.74 \mathrm{mmol}, 67 \%)$. Oil; $R_{\mathrm{f}}=0.33$ ( $n$-hexane $/ A c \mathrm{OEt}=75 /$ $25+1 \% \mathrm{MeOH}$ ); IR (film): $\bar{\nu}=3031,2988,2960,2906$, $2862,1955,1879,1741,1613,1566,1496,1478,1455$, $1368,1336,1310,1265,1229,1181,1141,1104,1030$, 1015, 788, 738, $699 \mathrm{~cm}^{-1} ;{ }^{1} \mathrm{H}$ NMR $\left(400 \mathrm{MHz}, \mathrm{CDCl}_{3}\right)$ : $\delta=7.40-7.28(\mathrm{~m}, P h), 6.15(\mathrm{~d}, J=3.1 \mathrm{~Hz}, \mathrm{H}-4), 6.04(\mathrm{~d}$, $J=3.1 \mathrm{~Hz}, \mathrm{H}-5), 4.56\left(\mathrm{~s}, \mathrm{CH}_{2}-P h\right), 4.20(\mathrm{q}, J=7.1 \mathrm{~Hz}$, $\left.\mathrm{H}-1^{1}\right), 3.74(\mathrm{t}, J=6.9 \mathrm{~Hz}, \mathrm{H}-8), 3.65(\mathrm{~s}, \mathrm{H}-2), 2.96(\mathrm{t}$, $J=6.9 \mathrm{~Hz}, \mathrm{H}-7), 1.29\left(\mathrm{t}, J=7.1 \mathrm{~Hz}, \mathrm{H}-1^{2}\right) \mathrm{ppm} ;{ }^{13} \mathrm{C} \mathrm{NMR}$ $\left(100 \mathrm{MHz}, \mathrm{CDCl}_{3}\right): \delta=170.0(C=\mathrm{O}), 153.0(\mathrm{C}-3), 146.6$ (C-6), 138.7 (1C, Ph), 128.8, 128.1, 128.0 (5C, Ph), 109.0 (C-4), 107.4 (C-5), $73.4\left(\mathrm{CH}_{2}-\mathrm{Ph}\right), 68.7(\mathrm{C}-8), 61.5\left(\mathrm{C}-1^{1}\right)$, 34.7 (C-2), 29.3 (C-7), $14.6\left(\mathrm{C}-1^{2}\right)$ ppm; ESI-MS: $m / z=312$ (10), $311\left(59,[\mathrm{M}+\mathrm{Na}]^{+}\right), 306\left(51,\left[\mathrm{M}+\mathrm{H}_{2} \mathrm{O}\right]^{+}\right), 290(21)$, $289\left(100,[\mathrm{M}+\mathrm{H}]^{+}\right), 215(12)$.

\section{Ethyl 2-(5-(2-(benzyloxy)propyl)furan-2-yl)acetate}

$\left(25, \mathrm{C}_{18} \mathrm{H}_{22} \mathrm{O}_{4}\right)$

General procedure with $\mathbf{1 5}(2.419 \mathrm{~g}, 5.42 \mathrm{mmol}), \quad \mathrm{LiCl}$ (706 mg, $16.65 \mathrm{mmol}$ ), and $\mathrm{H}_{2} \mathrm{O}(181 \mathrm{mg}, 10 \mathrm{mmol})$ in $10 \mathrm{~cm}^{3}$ $D M S O$ heated at $190^{\circ} \mathrm{C}$ for $2 \mathrm{~h}$. Chromatography afforded $25(1.035 \mathrm{~g}, 3.42 \mathrm{mmol}, 63 \%)$. Oil; $R_{\mathrm{f}}=0.36(n$-hexane/ $A c \mathrm{OEt}=75 / 25+1 \% \mathrm{MeOH}$ ); IR (film): $\bar{\nu}=3031,2977$, 2930, 2871, 1741, 1454, 1374, 1338, 1216, 1180, 1133, 1097, $1029,1014 \mathrm{~cm}^{-1} ;{ }^{1} \mathrm{H}$ NMR $\left(400 \mathrm{MHz}, \mathrm{CDCl}_{3}\right): \delta=7.40-7.27$ $(\mathrm{m}, P h), 6.16(\mathrm{~d}, J=3.0 \mathrm{~Hz}, \mathrm{H}-4), 6.05(\mathrm{~d}, J=3.0 \mathrm{~Hz}, \mathrm{H}-5)$, $4.57(\mathrm{~d}, J=11.8 \mathrm{~Hz}, \mathrm{CH} H-P h), 4.51(\mathrm{~d}, J=11.8 \mathrm{~Hz}, \mathrm{CHH}-$
$P h), 4.20$ (q, $J=7.1 \mathrm{~Hz}, \mathrm{H}-1^{1}$ ), 3.85 (sext, $J \approx 6.4 \mathrm{~Hz}, \mathrm{H}-8$ ), $3.66(\mathrm{~s}, \mathrm{H}-2), 2.96(\mathrm{dd}, J=14.9,6.3 \mathrm{~Hz}, \mathrm{H}-7 \mathrm{a}), 2.75(\mathrm{dd}$, $J=14.9,6.5 \mathrm{~Hz}, \mathrm{H}-7 \mathrm{~b}), 1.28\left(\mathrm{t}, J=7.1 \mathrm{~Hz}, \mathrm{H}-1^{2}\right), 1.26(\mathrm{~d}$, $J=6.3 \mathrm{~Hz}, \mathrm{H}-9) \mathrm{ppm} ;{ }^{13} \mathrm{C}$ NMR $\left(100 \mathrm{MHz}, \mathrm{CDCl}_{3}\right): \delta=$ $170.0(C=\mathrm{O}), 153.0(\mathrm{C}-3), 146.6(\mathrm{C}-6), 139.2,128.7,128.0$, 127.8 (6C, Ph), 109.1 (C-4), 108.3 (C-5), 74.3 (C-8), 71.1 $\left(\mathrm{CH}_{2}-\mathrm{Ph}\right), 61.5\left(\mathrm{C}-1^{1}\right), 35.8(\mathrm{C}-7), 34.7(\mathrm{C}-2), 20.2(\mathrm{C}-9)$, $14.6\left(\mathrm{C}-1^{2}\right) \mathrm{ppm}$; ESI-MS: $m / z=341$ (13), 326 (23), 325 $\left(100,[\mathrm{M}+\mathrm{Na}]^{+}\right), 324(25), 315(15)$.

Ethyl 2-(5-(2-acetoxy-ethyl)-furan-2-yl)acetate $\left(\mathbf{2 6}, \mathrm{C}_{12} \mathrm{H}_{16} \mathrm{O}_{5}\right)$ and Diethyl 2-(5-(2-acetoxyethyl)furan2-yl)malonate $\left(27, \mathrm{C}_{15} \mathrm{H}_{20} \mathrm{O}_{7}\right)$

General procedure with $\mathbf{1 6}(128 \mathrm{mg}, 0.33 \mathrm{mmol}), \quad \mathrm{LiCl}$ $(43.6 \mathrm{mg}, 1.03 \mathrm{mmol})$, and $\mathrm{H}_{2} \mathrm{O}(23.5 \mathrm{mg}, 1.31 \mathrm{mmol})$ in $1.35 \mathrm{~cm}^{3} D M S O$ heated at $160^{\circ} \mathrm{C}$ for $2 \mathrm{~h}$. According to NMR, $26(0.107 \mathrm{mmol}, 32 \%)$ and $27(0.056 \mathrm{mmol}, 17 \%)$ were afforded. They were partially separated by chromatography for characterization.

26: Oil; $R_{\mathrm{f}}=0.27(n$-hexane $/ A c \mathrm{OEt}=75 / 25+1 \% \mathrm{MeOH})$; ${ }^{1} \mathrm{H}$ NMR $\left(400 \mathrm{MHz}, \mathrm{CDCl}_{3}\right): \delta=6.14(\mathrm{~d}, J=3.1 \mathrm{~Hz}, \mathrm{H}-4)$, $6.04(\mathrm{~d}, J=3.1 \mathrm{~Hz}, \mathrm{H}-5), 4.31(\mathrm{t}, J=6.9 \mathrm{~Hz}, \mathrm{H}-8), 4.20(\mathrm{q}$, $\left.J=7.1 \mathrm{~Hz}, \mathrm{H}-1^{1}\right), 3.65(s, \mathrm{C}-2), 2.96(\mathrm{t}, J=6.9 \mathrm{~Hz}, \mathrm{H}-7), 2.07$ (s $\left(\mathrm{CH}_{3}-\mathrm{C}=\mathrm{O}\right), 1.29\left(\mathrm{t}, J=7.1 \mathrm{~Hz}, \mathrm{H}-1^{2}\right) \mathrm{ppm} ;{ }^{13} \mathrm{C}$ NMR $\left(100 \mathrm{MHz}, \mathrm{CDCl}_{3}\right): \delta=171.4\left(\mathrm{CH}_{3}-\mathrm{C}=\mathrm{O}\right), 169.9(\mathrm{C}=\mathrm{O})$, 151.7 (C-3), 147.1 (C-6), 109.1 (C-4), 107.7 (C-5), 62.7 (C-8), $61.5\left(\mathrm{C}-1^{1}\right), 34.6(\mathrm{C}-2), 28.1(\mathrm{C}-7), 21.3\left(\mathrm{CH}_{3}-\mathrm{C}=\mathrm{O}\right), 14.6$ $\left(\mathrm{C}-1^{2}\right)$ ppm; ESI-MS: $m / z=264\left(11,[\mathrm{M}+\mathrm{H}+\mathrm{Na}]^{+}\right), 263$ $\left(100,[\mathrm{M}+\mathrm{Na}]^{+}\right)$.

27: Oil; $R_{\mathrm{f}}=0.35(n$-hexane $/ A c \mathrm{OEt}=75 / 25+1 \% \mathrm{MeOH})$; ESI-MS: $m / z=351$ (52), $336(16), 335\left(100,[\mathrm{M}+\mathrm{Na}]^{+}\right)$.

Ethyl 2-(5-(2-acetoxyethyl)furan-2-yl)acetate (26, $\left.\mathrm{C}_{12} \mathrm{H}_{16} \mathrm{O}_{5}\right)$ and Ethyl 2-(5-(2-hydroxyethyl)furan2-yl)acetate $\left(19, \mathrm{C}_{10} \mathrm{H}_{14} \mathrm{O}_{4}\right)$

General procedure with $\mathbf{1 6}(2.85 \mathrm{~g}, 7.42 \mathrm{mmol}), \mathrm{LiCl}(960 \mathrm{mg}$, $22.6 \mathrm{mmol})$, and $\mathrm{H}_{2} \mathrm{O}(130 \mathrm{mg}, 7.22 \mathrm{mmol})$ in $19 \mathrm{~cm}^{3}$ DMSO heated at $190^{\circ} \mathrm{C}$ for $2 \mathrm{~h}$. Chromatography afforded $26(70.8 \mathrm{mg}$, $0.295 \mathrm{mmol}, 4 \%)$ and 19 (204.3 mg, $1.032 \mathrm{mmol}, 14 \%)$. Data for $\mathbf{2 6}$ were the same as above, data for $\mathbf{1 9}$ were the same as previously described by our group [4].

\section{Acknowledgements}

NMR Spectra $(400 \mathrm{MHz})$ were measured by Heinz Bursian and Dr. Claude Saturnin, mass spectra by Dr. Nicolas Mottier and Dr. Bernard Jean-Denis, CHN and HR-MS analyses were made in the Ecole d'ingénieurs et d'architectes de Fribourg. We thank the University of Neuchâtel and the Swiss National Science Foundation for financial support.

\section{References}

[1] Part I: Simone J-M, Loiseau F, Carcache D, Bobal P, Jeanneret-Gris J, Neier R (2007) Monatsh Chem preceding publication

[2] Buhlmann P, Pretsch, E, Bakker E (1998) Chem Rev 98: 1593 
[3] Pretsch E, Badertscher M, Welti M, Maruizumi T, Morf WE, Simon W (1988) Pure Appl Chem 60: 567

[4] Preceding paper on nonactic acid derivatives: Loiseau F, Simone J-M, Carcache D, Bobal P, Neier R (2007) Monatsh Chem

[5] a) Schneider M, Engel N, Boensmann H (1984) Angew Chem Int Ed 23: 66; b) Björkling F, Boutelje J, Gatenbeck S, Hult K, Norin T, Szmulik P (1985) Tetrahedron 41: 1347; c) Luyten M, Müller S, Herzog B, Keese R (1987) Helv Chim Acta 70: 1250; d) Ahmar M, Bloch R, Bortolussi M (1991) Synth Comm 21: 1071
[6] a) Ohno M, Otsuka M (1989) Org React 37: 1; b) Mohr M, Waespe-Sarcevic N, Tamm C, Gawronska K, Gawronski JK (1983) Helv Chim Acta 66: 2501

[7] a) Krapcho AP, Lovey AJ (1973) Tetrahedron Lett 12: 957; b) Krapcho AP (1982) Synthesis 805; c) Krapcho AP, Weimaster JF, Eldridge JM, Jahngen EGE, Lovey AJ, Stephens WP (1978) J Org Chem 43: 138

[8] Kruse CG, Janse ACV, Dert V, Van der Gen A (1979) J Org Chem 44: 2916

[9] Padgett HC, Csendes IG, Rapoport H (1979) J Org Chem 44: 3492 